\title{
Reform retractions to make them more transparent
}

\author{
Quan-Hoang Vuong \\ Nature \\ Volume 582, Issue 7811, p. 149 \\ doi:10.1038/d41586-020-01694-x \\ URL: https://www.nature.com/articles/d41586-020-01694-x

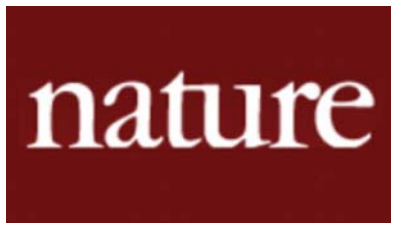

11 June 2020

*Reference:

Vuong, Q.-H. (2020). Reform retractions to make them more transparent. Nature, 582(7811), 149. 


\section{Reform retractions to make them more transparent}

\section{The scientific community should agree on the essential information to be provided when pulling a paper from the scientific literature.}

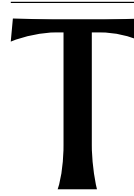

his month, both The New England Journal of Medicine and The Lancet retracted controversial studies testing COVID-19 treatments. Neither noted the part that public critique played. These are not the first papers and preprints related to the pandemic to be pulled, often with sparse explanation. The blog Retraction Watch has set up a tally.

Retractions are essential to keep the scientific literature trustworthy, but the notices for them are inconsistent and often uninformative. That prevents people from assessing papers and strategies to promote integrity; it also stigmatizes honest errors. Including more information could help.

Last year, I analysed more than 2,000 retraction notices culled from more than 20,000 listed on Retraction Watch and by major publishers (Q.-H. Vuong Learn. Publ. 33, 119-130;2020). A little more than half did not say who initiated the retraction. Around $10 \%$ gave no reason. Some simply read: "This paper has been retracted."

There have been many suggestions for improvements, including replacing retractions with post-publication amendments to the paper or with complicated taxonomies of errata, corrections, self-retractions, partial retractions, retractions with replacements, and refutations or removals. The rate of retractions has increased worldwide over the past few years, but none of these ideas has caught on.

A more manageable (perhaps intermediate) step would be to outline four pieces of information that should be provided with any retraction. Specifically: who initiated it; the cause (such as severe errors, plagiarism or fraudulent practices); whether there is consensus between editors and authors about it; and whether post-publication review (such as comments on PubPeer) was involved. These steps would, in my opinion, prompt researchers and institutions to encourage retractions when warranted.

Right now, stigma keeps researchers from admitting their mistakes. Yet multiple examples show that researchers who act to correct mistakes are lauded rather than shamed. Witness the reaction this year to the retraction of a paper on using enzymes in chemical synthesis by Nobel-prizewinning chemist Frances Arnold. The retraction notice identified the cause and initiator, allowing readers to appreciate the honesty and self-correcting spirit of a world-renowned team of scientists. If such transparency were routine, it might ease the pain of retraction, and increase the public's understanding of how science works.

It is more than a decade since the first formal call for retractions to be more informative. In 2009, the

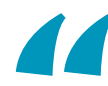

\section{Retractions}

arenot

intrinsically

bad: they are

a practical

way to

correct

for human

fallibility."

\section{Quan-Hoang}

Vuong is director of the Centre for Interdisciplinary Social Research, Phenikaa University, Hanoi, Vietnam. e-mail: hoang. vuongquan@ phenikaa-uni.edu.vn
Committee on Publication Ethics (COPE) issued guidelines recommending, among other things, that publishers state who is retracting the article and why. Retraction Watch issued a similar wishlist in 2015. My analysis, which looked at notices from 1975 to 2019, was unable to detect any change in practice since COPE issued its guidelines, because nearly $97 \%$ of the retractions in my sample were from 2009 or later. But it is safe to say that publishers fall short of the ideals. Perhaps a simple format would be easiest to adopt.

Even so, implementing the four-component retraction notice would be difficult. The second component, stating a cause, is probably the most tricky. Although editors decide whether to retract a paper, it is usually research institutions that investigate whether misconduct has occurred. These investigations are often slow and inconclusive, so journals often substitute safer, blander terms such as error, loss of data or replication failure. A publication-integrity checklist published earlier this year might help: it is a series of questions such as whether a study could plausibly be done as described, and could be used to pinpoint problems without requiring a formal declaration of misconduct (A. Grey et al. Nature 577, 167-169; 2020).

Here, the third component of the retraction notice comes in. This makes clear that editors and authors can provide different reasons for the retraction, rather than having to agree on the final wording.

The fourth component stresses the role of the users of research in safeguarding the literature, and gives credit for this process. The people who flagged problems can stay unidentified if they wish, and editors or institutions should explain what they did to investigate.

I think reform in retraction processing would promote best practice. Four-component notices would offer publishing norms, especially for emerging economies. And transparency could allow researchers and editors everywhere to learn about mistakes and misconduct that warrant retractions, as well as the grey areas of disagreement.

Furthermore,journals should mandate that every paper carry a section about its limitations that is free to read, like an abstract. A formal statement of shortcomings should keep authors and the public from overstating a study's claims, but fewer than $9 \%$ of papers in my analysis had such a section. Making such disclosure mandatory could prevent embarrassment and, more importantly, help to reverse the idea that studies that seem 'conclusive' or 'clean' are more important than transparent research narratives.

Retractions expose flaws and possibly misconduct in research, but also show that the error-detection mechanism is working well. With this understanding, we must rehabilitate the term. Retractions are not intrinsically bad: they are a practical way to correct for human fallibility and strengthen the scientific enterprise. 